\title{
SKIN SEGMENTATION USING DIFFERENT INTEGRATED COLOR MODEL APPROACHES FOR FACE DETECTION
}

\author{
Mrunmayee V. Daithankar ${ }^{1}$, Kailash J. Karande ${ }^{2}$ \\ ${ }^{1}$ ME Student, Electronics and Telecommunication Engineering Department, SKN Sinhgad College of Engineering, \\ Pandharpur, Maharashtra, India \\ ${ }^{2}$ Principal, Electronics and Telecommunication Engineering Department, SKN Sinhgad College of Engineering, \\ Pandharpur, Maharashtra, India
}

\begin{abstract}
This paper presents an efficient skin segmentation algorithm which depends on integration of different color models. Among different low level features such as edge, shape, color, texture; color processing is computationally fast and easy to implement. Color constancy is the ability of human vision system to resolve object colors in a scene independent of the illuminant. Skin color is one of the powerful and effective features to detect the face. To represent skin color different color models are presents which are having luminance and chrominance components like $\mathrm{YCbCr}, \mathrm{YCgCr}$ and HSI. Though geographically, people of different country has different skin color. This difference lies in their intensity not on their chrominance. Study and Analysis of these color models motivates us to combine the uncommon features of these color models for efficient skin segmentation. As the skin color is highly affected by illumination changes due to luminance i.e. brightness part of color models, we have avoided this component in feature's combination. Integration of color models contains combination of only chrominance components which will be helpful in handling illumination affects. After skin segmentation, morphological operations such as dilation which adds pixels to the boundary of objects in image and erosion which removes pixels from object's boundaries are used to get more smooth results. To locate face, face region is detected by checking either 4-point or 8-point connectivity as per requirement and bounded by a rectangle. This process gives us efficient face detection system with $100 \%$ face detection rate. This efficient face detection system may results in better final system such as face recognition system, human computer interface system, video surveillance, etc. where face detection is initial and important step.
\end{abstract}

Keywords: Skin Segmentation, Color Models, Integration of color models, Face Detection, Face localization

\section{INTRODUCTION}

Improvement at the stage of detection of face region from images becomes necessary to reach at higher efficient final systems such as video surveillance systems, face recognition systems, HCI systems, etc. where face detection is first and important step. This necessity leads to active research area of biometrics for researchers. Different techniques used for face detection with advantages and disadvantages now become scope for advancement to achieve efficiency in locating face region perfectly. Knowledge based, feature based, template matching based and appearance based methods with different techniques are used for face detection. The comparative analysis of these methods is done by Xiaobo Zhu et al [1], overview is given below:

\section{- Knowledge Based Methods:}

Knowledge-based methods use the knowledge about a basic face, such as the ellipse shape and the triangle feature, to obtain the final region of a face. Knowledge based methods can greatly reduce computational cost, but they are rotationdependent.

\section{- Template Matching Based Methods:}

Template matching based methods use the similarity between an input image and the template. These methods are easy to implement, but they are scale-dependent, rotation-dependent, and computationally expensive.

\section{- Appearance Based Methods:}

Appearance based or machine learning-based methods have high detection rates, but their accuracy depends on the training samples. Most of these methods focus on frontal faces or faces with fixed orientations.

\section{- Feature Based Methods:}

Feature-based methods use low level features (such as color, edge, shape, and texture) to detect faces. Feature based methods are scale independent, rotation independent, and fast. Many authors found that skin color, which is scale independent and rotation independent, can greatly reduce computational cost of face detection.

Face detection is challenging area of research due to the variability of human face with change in skin color, expression, illumination changes, occlusion and pose variation. The processing of color which comes under 
feature based method is more advantageous as compare to other low level facial feature analysis [2]. Fattah Alizadeh et al [3] explain the two approaches for face detection using skin color. First approach is Pixel-Based Model, which is based on processing the pixels for all parts of the human skin color. In this approach each pixel is processed independently, and its final status, i.e. it is skin color or not, will be determined. Then, based on facial structure or other options, it will be decided that set of points that were marked as skin, it belong to face or not.

The second approach is based on the status of region of the image. In this approach at first, the necessary attempt is done to segregate the region that may produce a face within the given image. And then using the previous information and knowledge it will be decided that specifies if the region belongs to face or not.

\subsection{Skin Color Segmentation}

Among various low facial features such as edge, shape, skin color and texture; skin color is prominent tool for extracting face region due to its fast processing and ease of implementation. Although color processing is advantageous but sensitive to following conditions which are discussed by Ukil Yan et al. [4] and Nidhi Tiwari et al. [5]:

- Illumination conditions: A change in the spectral distribution and the illumination level of light source (indoor, outdoor, highlights, shadows, color temperature of lights)

- Camera characteristics: The color reproduced by a CCD camera is dependent on not only the illumination conditions but also the spectral sensitivities of a camera sensor.

- Ethnicity: Skin color varies according to ethnic groups.

- Individual characteristics: Individual characteristics such as age, sex and body parts affect the skin color.

- Other factors: Different factors such as makeup, glasses, background colors, shadows and motion affect the skin color.

The skin color is defined by different color models like RGB, CMY, YUV, YIQ, YPbPr, YCbCr, YCgCr, YDbDr, HSV and CIE-XYZ. Comparative study and analysis of these models is done by Jose M. Chaves-Gonzalez et al. [6] and Manuel et al. [7]. The results of this study and analysis tell us that $\mathrm{YCbCr}, \mathrm{YCgCr}$ and $\mathrm{HSI}$ models gives most promising results for skin segmentation and becomes most popular among others. Introduction to these color models is given under next point.

\subsection{Color Models}

\subsubsection{YCbCr Model:}

In this color model, Y represents luminance component i.e. light intensity and $\mathrm{Cb}, \mathrm{Cr}$ represents blue difference of the chromaticity component and red difference of the chromaticity component respectively. The chrominance components are not depends on luminance component [8].

\subsubsection{YCgCr Model:}

In the $\mathrm{YCgCr}$ color space, a human skin color model can be concentrated in a small region of the $\mathrm{Cg}-\mathrm{Cr}$ plane. This color space includes information about green difference instead of blue difference, which can be more useful for skin color detection [9].

\subsubsection{HSI Model:}

In this color model, H-Hue describes the main color i.e. depth of color, S-saturation gives purity of the color and IIntensity indicates the brightness of the shade. HSI color model has been used for image processing because it can separate the chromaticity from the intensity of the image [10].

So our interest is in combining features of these color models to get efficient face detection system.

\section{FLOW OF PROPOSED WORK}

The flow for our proposed work is given in Fig.1. The first step of dissertation is to take RGB image as input to system. This image is pre-processed by converting from RGB to appropriate color models. After this conversion, we have segmented image in two parts as skin region and non skin region by applying thresholds for each channel of model. The threshold values come from experimentation of histograms. Thus skin region is segmented. For smooth skin area, morphological operations such as erosion and dilation are used.

The 4-point and 8-point connectivity is checked on white pixels to segment face region from image. To bound face in image with rectangle, height to width ratio is applied. This ratio avoids false detections. At last, image of face with bounding box is displayed.

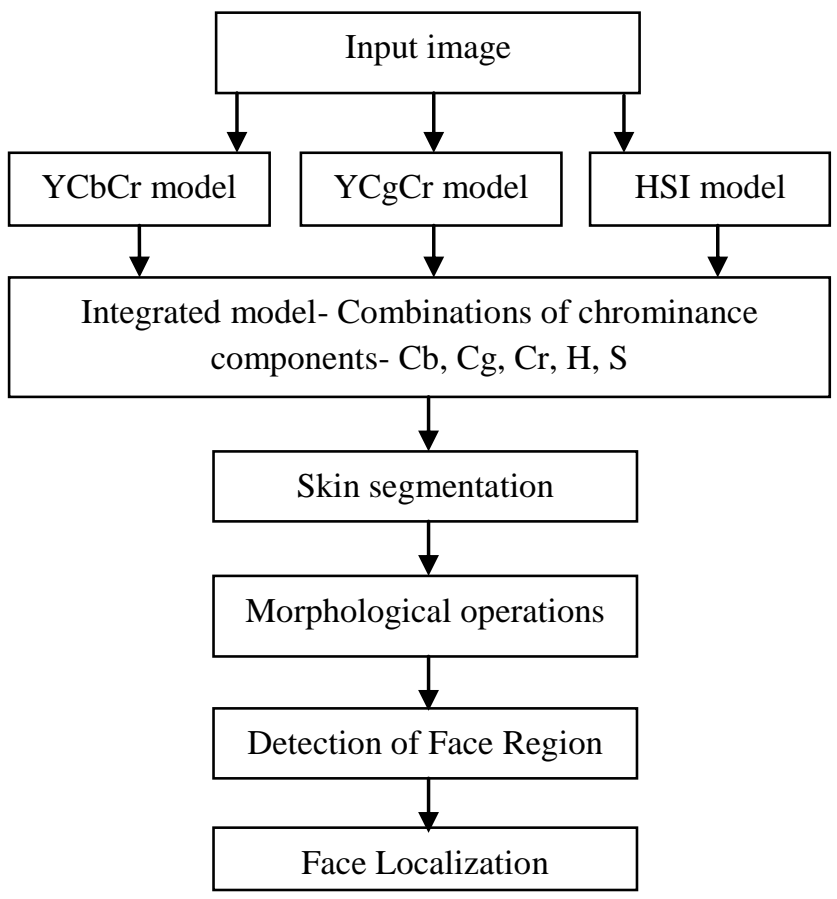

Fig-1 Flow of proposed work 


\subsection{Integration of Color Models}

Different combinations of chrominance components of most popular color models and their threshold values which are used for skin segmentation, shown in Table -1. These threshold values are calculated from histogram processing.

Table -1: Integrated color models with threshold values

\begin{tabular}{|c|c|c|}
\hline $\begin{array}{l}\text { Combination } \\
\text { of color models }\end{array}$ & $\begin{array}{l}\text { Proposed } \\
\text { integrated models }\end{array}$ & $\begin{array}{l}\text { Threshold values } \\
\text { for each channel }\end{array}$ \\
\hline $\begin{array}{lr}1] & \mathrm{YCbCr}, \\
\mathrm{YCgCr} & \text { and } \\
\text { HSI } & \end{array}$ & 1] $\mathrm{HSCbCgCr}$ & $\begin{array}{l}0.4<\mathrm{H}<0.8, \\
0.4<\mathrm{S}<0.6, \\
82<\mathrm{Cb}<160, \\
110<\mathrm{Cg}<125, \\
125<\mathrm{Cr}<165\end{array}$ \\
\hline $\begin{array}{l}\text { 2] } \mathrm{YCgCr} \text { and } \\
\text { HSI }\end{array}$ & $\begin{array}{l}\text { 1] } \mathrm{HSCgCr} \\
\text { 2] } \mathrm{HCgCr}\end{array}$ & $\begin{array}{l}0.4<\mathrm{H}<0.8 \\
0.4<\mathrm{S}<0.6 \\
110<\mathrm{Cg}<125, \\
125<\mathrm{Cr}<130\end{array}$ \\
\hline $\begin{array}{l}\text { 3] } \mathrm{YCbCr} \text { and } \\
\mathrm{YCgCr}\end{array}$ & 1] $\mathrm{CbCgCr}$ & $\begin{array}{l}82<\mathrm{Cb}<160, \\
110<\mathrm{Cg}<125, \\
125<\mathrm{Cr}<165\end{array}$ \\
\hline $\begin{array}{l}\text { 4] } \mathrm{YCbCr} \text { and } \\
\text { HSI }\end{array}$ & $\begin{array}{l}\text { 1] } \mathrm{HSCbCr} \\
\text { 2] } \mathrm{HCbCr}\end{array}$ & $\begin{array}{l}0.4<\mathrm{H}<0.8, \\
0.12<\mathrm{S}<0.3, \\
82<\mathrm{Cb}<160, \\
125<\mathrm{Cr}<165\end{array}$ \\
\hline
\end{tabular}

\section{EXPERIMENTAL RESULTS /DISCUSSIONS}

The integrated color models are implemented with the help of MATLAB software. The experimentation is carried out on randomly collected 50 images from internet. These images are with different backgrounds, various pose and illumination changes. Each designed integrated model for skin segmentation is compared to get best combination of chrominance components.

Due to illumination changes and complex background, some non-skin area also marked as skin region by white pixels. The segmented skin area becomes smoother when we apply morphological operations like dilation and erosion on image. After that, checking of white pixels connectivity either by 4-point or 8-point and applying height to width ratio, we reached to efficient face region detection and localization.

\subsection{Results of $\mathrm{HSCbCgCr}$ Color Model}

The $\mathrm{HSCbCgCr}$ integrated color model is combination of $\mathrm{YCbCr}, \mathrm{YCgCr}$ and HSI color models. The RGB input image is first transferred in these three color models and using threshold values from Table-1, skin region is segmented to locate face area. The face detection rate of this model is $84 \%$. This process is shown in Fig-2:

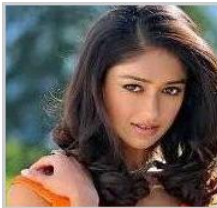

(a) Input image

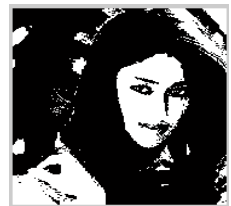

(b) Skin segmentation (c) Face detection

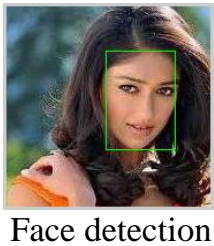

Fig-2 Implementation of $\mathrm{HSCbCgCr}$ integrated color model

\subsection{Results of $\mathrm{HSCgCr}$ Color Model:}

This is integration of chrominance components of HSI and $\mathrm{YCgCr}$ color models. Hue and saturation are taken from HSI model while green and red chrominance components are part of $\mathrm{YCgCr}$ model. The skin segmentation and face detection results of $\mathrm{HSCgCr}$ are shown in Fig-3. The face detection rate is $84 \%$.

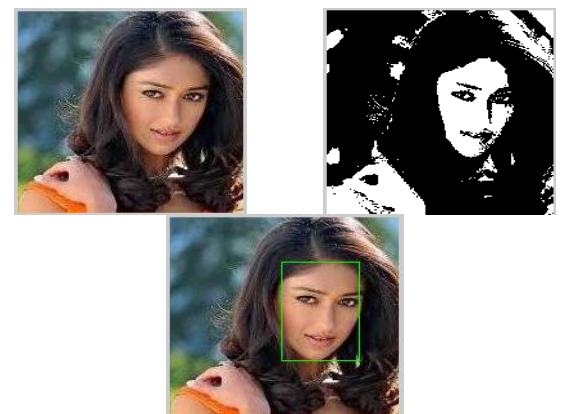

(a) Input image (b) Skin segmentation (c) Face detection

Fig-3 Implementation of $\mathrm{HSCgCr}$ integrated color model

\subsection{Results of $\mathrm{HCgCr}$ Color Model:}

The implementation of integrated model $\mathrm{HCgCr}$ is done with the help of combination of $\mathrm{YCgCr}$ and $\mathrm{HSI}$ color models. The avoidance of saturation component does not affect on efficiency of face detection. It has same face detection rate as of previous one i.e. $84 \%$. This implementation is shown in Fig-4:

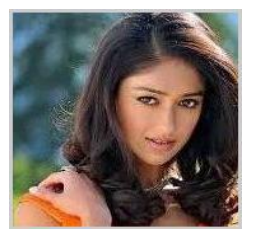

(a) Input image

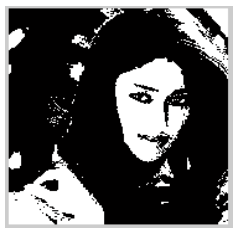

(b) Skin segmentation (c) Face detection

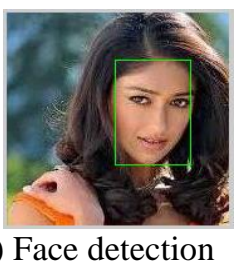

Fig-4 Implementation of $\mathrm{HSCgCr}$ integrated color model

\subsection{Results of $\mathrm{CbCgCr}$ Color Model:}

The Combination of $\mathrm{YCbCr}$ and $\mathrm{YCgCr}$ resulted in an integrated approach $\mathrm{CbCgCr}$. Threshold values are taken from Table-1. Fig-5 shows skin segmentation and face detection results. $\mathrm{CbCgCr}$ got $84 \%$ efficiency in face detection. 


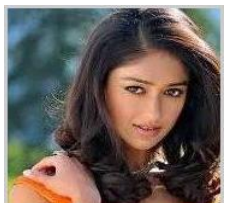

(a) Input image

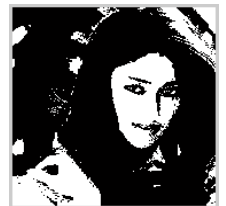

(b) Skin segmentation (c) Face detection

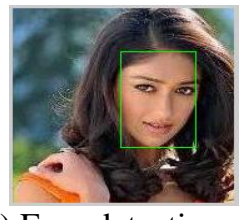

Fig-5 Implementation of $\mathrm{CbCgCr}$ integrated color model 3.5 Results of HSCbCr Color Model:

The color models $\mathrm{YCbCr}$ and HSI are combined together and implemented this integrated approach whose face detection rate is $90 \%$. These results are efficient than previously discussed models. The skin segmented and face detected result for this model is shown in Fig.6:

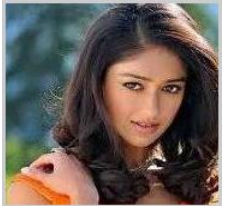

(a) Input image

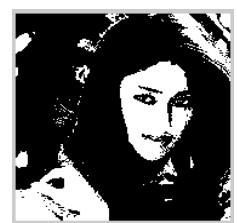

(b) Skin segmentation

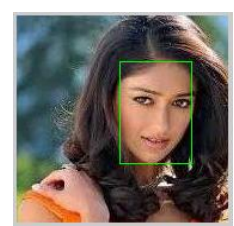

(c) Face detection
Fig-6 Implementation of HSCbCr integrated color model

\subsection{Results of $\mathrm{HCbCr}$ Color Model:}

This integrated approach is also implemented by combining $\mathrm{YCbCr}$ and HSI color models but the difference between previous model and $\mathrm{HCbCr}$ is avoidance of saturation component. Thus, skin segmentation is smoother than other implemented integrated approaches. This is resulted in more efficient face detection with $100 \%$ face detection rate. The skin segmented and face detected results with this model are shown in Fig-7:
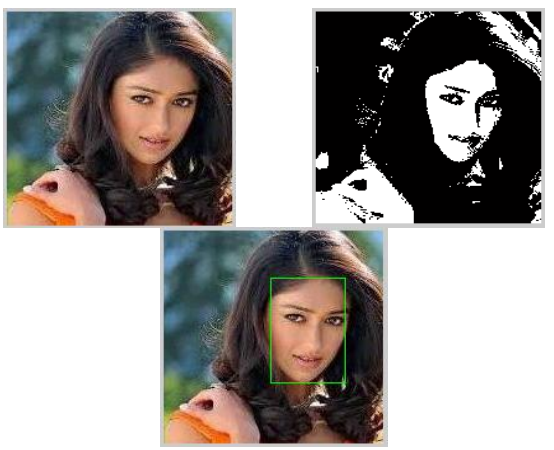
(a) Input image
(b) Skin segmentation
(c) Face detection

Fig-7 Implementation of $\mathrm{HCbCr}$ integrated color model

Table-2: Face detection rate for integrated models

\begin{tabular}{|l|l|}
\hline $\begin{array}{l}\text { Integrated color } \\
\text { models }\end{array}$ & Face detection rate \\
\hline 1] $\mathrm{HSCbCgCr}$ & $84 \%$ \\
\hline 2] $\mathrm{HSCgCr}$ & $84 \%$ \\
\hline 3] $\mathrm{HCgCr}$ & $84 \%$ \\
\hline 4] $\mathrm{CbCgCr}$ & $84 \%$ \\
\hline 5] $\mathrm{HSCbCr}$ & $90 \%$ \\
\hline $\mathbf{6}] \mathbf{H C b C r}$ & $\mathbf{1 0 0 \%}$ \\
\hline
\end{tabular}

More results of integrated approaches with the help of images are shown in Table-3.

Table-3: Results for integrated approaches

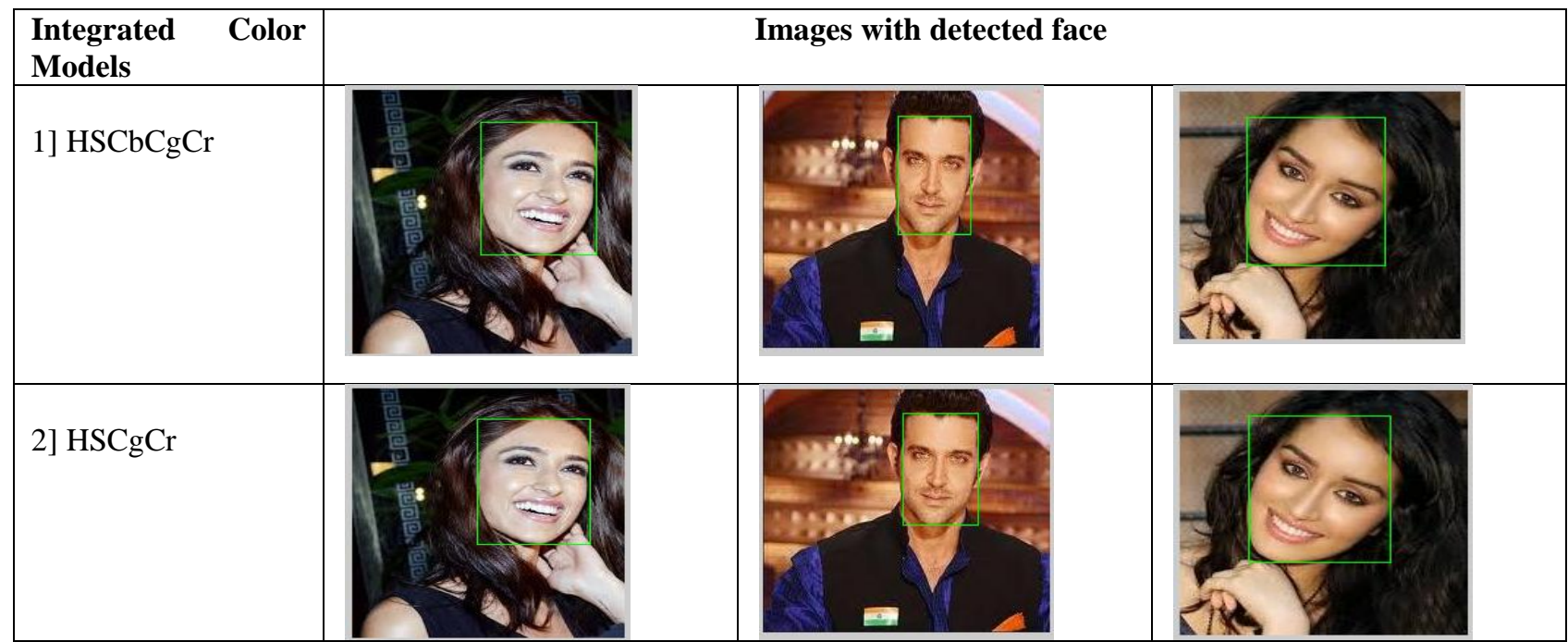




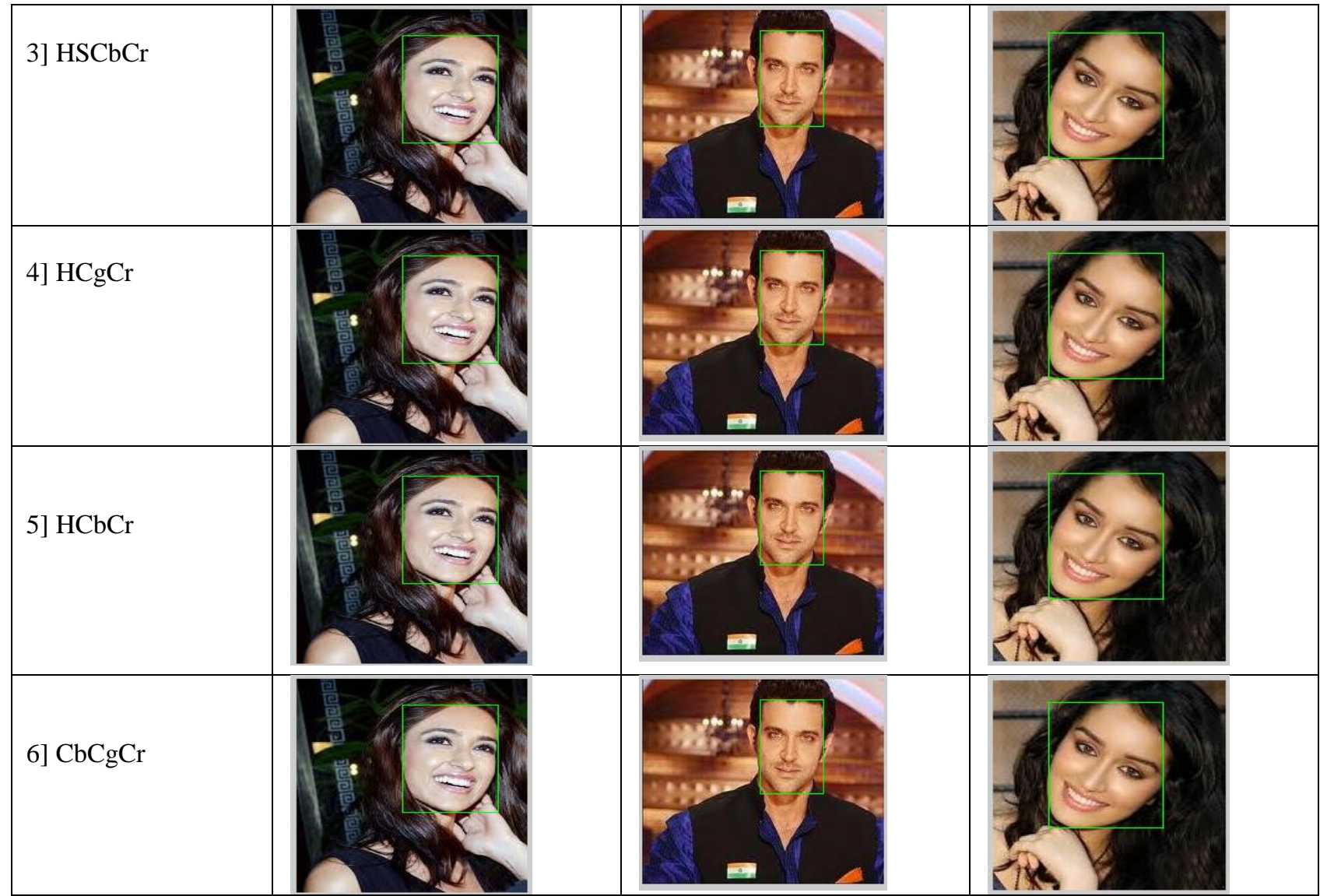

\section{CONCLUSIONS}

This paper gives new skin segmentation algorithm based on integration of different skin color models. The integrated approaches are combination of uncommon features of $\mathrm{YCbCr}, \mathrm{YCgCr}$ and HSI color models. The experimental results shows that, combination of green chrominance channel of $\mathrm{YCgCr}$ model with other channels of both YCbCr and HSI models does not provide satisfactory results. Integration of $\mathrm{YCbCr}$ and HSI color models gives better results than other combinations. Avoidance of saturation component shows smoother skin segmentation in $\mathrm{HCbCr}$ model whose face detection rate is $100 \%$. This algorithm provides robustness and efficiency than surveyed algorithms.

\section{REFERENCES}

[1] Xiaobo Zhu, Dong Ren, Zhenyu Jing, Lin Yan and Shuanghui Lei, "Comparative Research of the Common Face Detection Methods," IEEE 2nd International Conference on Computer Science and Network Technology, pp.1528-1533, 2012.

[2] Hewa Majeed Zangana and Imad Fakhri A Shaikhli, "A new algorithm for human face detection using skin color tone," IOSR Journal of Computer Engineering, vol.11, issue.6, pp. 31-38, June 2013.

[3] Fattah Alizadeh, Saeed Nalousi and Chiman Savari, "Face Detection in Color Images using Color Features of Skin," World Academy of Science, Engineering and Technology52, pp.223-229, 2011.
[4] Ukil Yang, Bongjoe Kim and Kwanghoon Sohn, "Illumination Invariant Skin Color Segmentation," ICIEA IEEE, pp. 636-641, 2009.

[5] Nidhi Tiwari, N. K. Mittal and S. G. Kerhalker, "Automatic face detection in frontal face color images," International Journal of Scientific Engineering and Technology, vol. 2, issue.7, pp. 670-674, July 2013.

[6] Jose M. Chaves-González , Miguel A. VegaRodríguez, Juan A. Gómez-Pulido and Juan M. Sánchez-Pérez, "Detecting skin in face recognition systems: A color spaces study," Digital Signal Processing, vol.20, pp. 806-823, 2010.

[7] Manuel C. Sanchez-Cuevas, Ruth M. Aguilar-Ponce and J. Luis Tecpanecatl- Xihuitl, "A Comparison of Color Models for Color Face Segmentation," Procedia Technology, vol.7, pp.134 -141, 2013.

[8] Kailash J. Karande, "Localized Spatiotemporal Modular ICA for Face Recognition," IEEE Symposium on Computational Intelligence in Biometrics and Identity Management (CIBIM), pp.66-70, April 2013.

[9] Kamarul Hawari Bin Ghazali, Jie Ma, Rui Xiao and Solly Aryza lubis, "An innovative face detection based on $\mathrm{YCgCr}$ color space," International conference on solid state devices and materials science, vol.25, pp.2116-2124, 2012.

[10] Yogesh Tayal, Ruchika Lamba and Subhransu Padhee, "Automatic face detection using color based segmentation," International Journal of Scientific 
and Research Publications, vol.2, issue.6, pp.1-7, June 2012.

\section{BIOGRAPHIES}

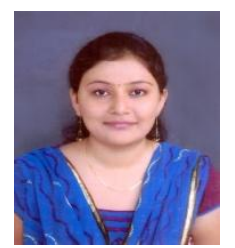

Mrunmayee V. Daithankar has completed B.E. (Electronics and Telecommunication Engineering) from SVERI's college of engineering, Pandharpur. India. Now she is student of ME -Electronics in SKN Pandharpur, India. Sinhgad College of Engineering,

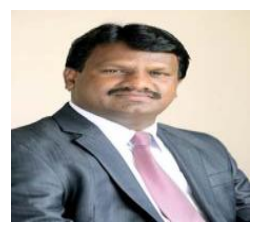

Dr.Karande Kailash Jagannath has completed his Ph.D in Electronics and Telecommunication from S. R.T.M University, Nanded, India. He has total 18 Publications in International and National Journals and Conferences. He is member of ISTE and IETE. Currently he is working as Principal at SKN Sinhgad College of Engineering, Pandharpur, India. 